\title{
Application of Multimedia Technology in Database and IT Service Management
}

\author{
Onimode Bayo Mohammed \\ Computer Science Department \\ Federal University of Technology, Minna \\ Niger State, Nigeria
}

\begin{abstract}
Multimedia is a blend of two or more media such as text, sound, graphics, animation and video, to effectually converse ideas to the users and this could be in linear or non-linear form [7]. The application of multimedia technology to database and IT service management has improved enormously in recent times. Multimedia system is a distinctive application which is of time-critical in its use in the various areas of computing. The spatial, secular, storage, processing, recovery, grouping and management requirements of data vary momentously from those that are applied for traditional data [2]. Therefore, the purpose of multimedia technology in database management system and in proper management of IT services to clients is to permit for an efficient way of performing these tasks in all its varied forms in an efficient form. A multimedia database management system affords an efficient storage and manoeuvring of multimedia technologies in all its diverse types. Database technology has offered means to store and recover high volumes of data in the various business domains [8]. Although, database systems have always been planned for the administration of alphanumeric data such as names and numbers, the basic nature of multimedia data are also considered and thus highlights the need for multimedia enhanced database management systems, and present the various obligations and issues required for developing such systems. The various areas of applications consist of, but not limited to digital libraries (text documents, images, sound, video etc), art and entertainment, content management, journalism etc [1]. For technology purposes, multimedia is a computer-based systems that apply associative relationships to allow the users of such systems to navigate and retrieve various information that are stored in a location which could be a combination of text, sounds, graphics, video, and other media formats [12].
\end{abstract}

\section{Keywords}

Multimedia, Technology, Database systems, metadata, management, IT service management, Object-oriented.

\section{INTRODUCTION}

Multimedia database management system is at the heart of multimedia information systems. Conventionally, a database consists of a managed collection of related data that are related to a given entity, whereas a database management system, or DBMS, is a collection of data that are interrelated with the set of programs that are applied to classify, produce, store, access, run, and possibly query the database for use. Equally, multimedia enhanced database can be viewed as a organized collection of multimedia data items, such as text, images, audio, graphic items, video and sketches [3]. Thus a multimedia DBMS provides support for several multimedia data types, and in addition services for the creation, access, query, storage and control of the multimedia database. The diverse data types that are involved in multimedia databases may necessitate unique techniques for the optimal access, indexing, storage and retrieval [5]. The multimedia DBMS ought to contain these unique requirements by offering highlevel ideas to handle the different data types, along with a suitable interface for their management. The composition and character of multimedia data from the various perspectives is also looked into [11]. These perspectives includes: excess information, spatial and sequential characteristics, shortage of textual depictions, array of data types, and the massive volumes of data that are applied. The combination of multimedia data types from unique but several sources exclusively characterizes multimedia information systems. Prior to detailing the abilities that are expected of a multimedia DBMS and the obligations that such systems should meet, consideration is first given to the attributes and the nature of multimedia information we enhances it usage and applications. Also application of multimedia technologies in the management of IT services is also discussed [6].

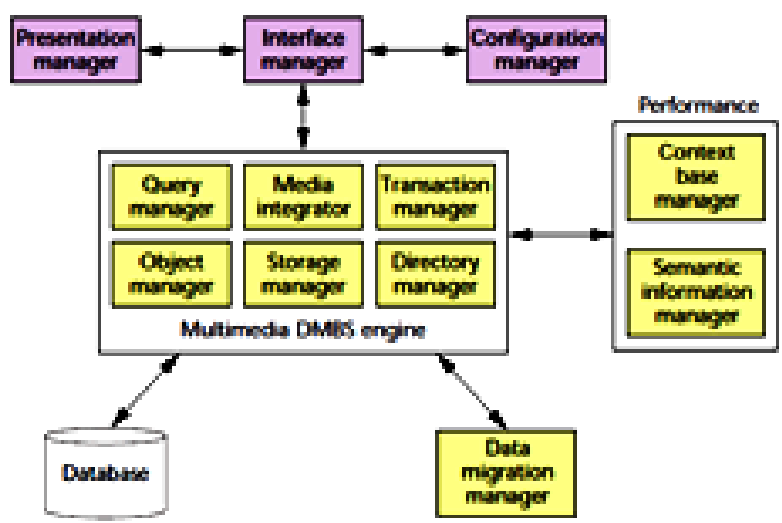

Fig.1: A high level architecture for a multimedia DBMS that meets the requirement for multimedia data.

\section{MULTIMEDIA DATABASES 2.1 Multimedia Data:}

There exist a couple of data types that can be described as multimedia data types. These are classic elements that are applies as the building mass of the hub generalized multimedia platforms, environments, or the integrating tools used. Thus the basic data types found in a typical multimedia database includes [1]: 
a) Text: the form in which the text can be stored can vary greatly;

b) Audio: is generated from an auditory recording device;

c) Images: black and white, coloured, maps, photographs, and paintings;

d) Graphic objects: ordinary drawings, sketches, and illustrations, or 3D objects;

e) Animation sequences: images or graphic objects which are usually independently generated;

f) Video: are also strings of images, called frames, but are typically recording a real-life event and are usually produced by a video recorder; and

g) Composite multimedia: is formed from a combination of two or more of the above data types, such as an blend of audio and video with a textual explanation.

Various multimedia data types like audio, video, and animation sequences also have chronological requirements, which have inferences on their storage, management, as well as their presentation. The exertions become more heightened when various data types from perhaps unrelated sources must be offered within or at a given time. Likewise, graphics, images, and video data have spatial limits in terms of their content [14]. Usually, individual objects in an image or a video frame have some spatial correlation between them. Such correlations typically produce some limits when searching for objects in a database. Large volumes of data also characterize multimedia information. For example, to store an uncompressed image of $1024 \times 728$ pixels at 24 bits per pixel involves a storage capacity of about $2 \mathrm{mb}$. With a compression ratio of 20:1, the storage requisite could be condensed to about $0.1 \mathrm{mb}$. Thus considering an instance for video, a 10-minute sequence of the same image at 30frames per second needs about $38,000 \mathrm{mb}$ of storage, which can be condensed to about $380 \mathrm{mb}$ using a compression ratio of $100: 1$ [6].

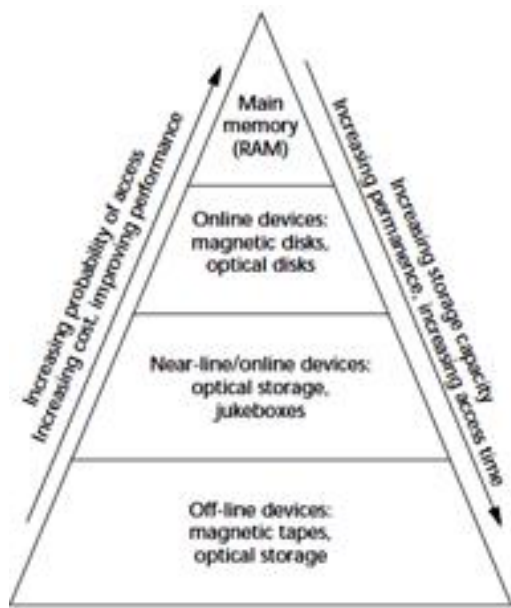

Fig. 2: A hierarchically storage for multimedia database

The potential for large volumes of data involved in multimedia information systems becomes obvious when considering for example, that a motion picture could run as long as two hours, and classic video ordnance would accommodate thousands of movies. An old adage says that a picture is worth more than a thousand words [1]. However, representing multimedia information as pictures or image series poses some hitches for information recovery due to the restraints of textual depictions of a multimedia practice and the enormous information accessible from it. The prospective information excess means that users may find it hard to make exact demands during information recovery. The inadequacies of textual narratives also imply the necessity for content-based access to multimedia information. Users need multiple nods (such as colour, shape, and texture) that are relevant to the multimedia content [13]

An additional attribute of multimedia information is that of it interface with such information types usually involves longperiod functions (such as with video data), with bits of more than a single client (as is typical in joint support settings). Conversely, in concerted settings, it is likely that the majority of multimedia data are likely to be accessed in a read only form. This assumption can be used to facilitate the provision of concurrency control algorithms.

\subsection{How Multimedia Technology is differently applied in Database}

Theoretically it ought to be feasible to treat multimedia database in the equal manner as data that based on the data types (for example: dates, numbers and characters), nevertheless, there exist some challenges that occurs as a result multimedia data as described in [17], these challenges are:

a. In case of audio and video databases, the time to retrieve information may be very crucial for example, video on order.

b. Multimedia data is large and thus affects the storage, processing, retrieval and transmission of multimedia data as contained in the database.

c. The substance of multimedia data are frequently captured with diverse "capture" procedures (for example, image processing) that may be rather unreliable. Multimedia processing techniques need to be able to handle diverse ways of content capture including manual ways and or automated methods.

d. Queries created by the user in the multimedia databases repeatedly cannot return with a textual answer. Slightly, the responses to a query may be a complex multimedia presentation that the user can look through at his/her spare time. Thus various outlines show how queries to multimedia databases may be utilised to create multimedia presentations that meet user's queries [1].

e. In conventional databases, user explicitly submits the point values of the objects inserted into the database; this is an automatic feature extraction and Indexing mode.

f. Comparatively, utilizing advanced tools such as image processing and pattern recognition tools for images, to extract the various elements and content of multimedia objects. As the size of data becomes very large, there is need for special data structures to be used for storing and indexing of such data.

\subsection{Basic Approaches for Data Retrieval}

Database management has a much stretched history and lots of approaches have been created to manage and query diverse database types in the database management systems. As given in [10], the fundamental approaches being applied for database management can be categorized into the following types: 
* The conventional database system: This is the broadly-used method to manage and search through a database for structured data. All the data in a database system thus must obey the rules of some predefined structures and checks (that is, the schemas). To prepare a database query, the user of such database must give which data objects are to be retrieved, the database tables from which they are to be extracted and predicate on which the retrieval is based. A query language for the database will generally be of the artificial kind, one with controlled syntax and vocabulary, such as the SQL.

* The Information Retrieval (IR) system: the IR system is majorly used to search large text collections in a database, in which the text of the data is described by an indexer using keywords or a textual abstract, and keywords or natural language is used to express query demands. For example for an image or video we have to describe it in words or in a way need to store lot of metadata in textual form [4].

* The Content Based Retrieval (CBR) system: This approach is used to retrieve desired multimedia objects from a large group on the basis of the features (such as texture, shape, colour and, etc.) of the database that can be automatically extracted from the objects themselves. Although keyword can be treated as a "feature" for text data, traditional information retrieval has much more higher performance than content-based retrieval because keyword has the proven ability to represent semantics, while no features have shown convincing semantic describing ability. But major drawback of this method is that it lacks precision.

* The Graph or tree pattern matching: This approach aims to retrieve object sub-graphs from an object graph according to some denoted patterns specified.

\subsection{Metadata}

Metadata are data about data. The term "meta" comes from a Greek word, denoting something of a higher or more fundamental nature. Generally speaking, metadata can refer to any data that are used to describe the content, condition, quality, and other aspects of data for humans or machines to be able to locate, access and understand the data contained. Thus, metadata information can help the users to get a synopsis of the data [3].

\section{Why do we need Metadata?}

When an image tells nothing about itself except the plain fact that it is a picture of an object, without reading the associated metadata, it is not possible for a user to discern the properties of the image such as who snapped the picture, when and where it was taken, what is the resolution of the picture amongst others, all of which are very vital information that helps to determine the appropriateness of the image for a certain application before the user takes a look at the actual data. Metadata thus plays far more significant role in the management of multimedia data than does the running of traditional well-structured data. Some of the reasons as described by [9] include:

* Different Query Paradigm: The exact-match idea for querying is no longer apt or sufficient for querying or retrieving various types of digital data.

* Inadequate Processing Technique: Content-based processing techniques are excessively hard to analyze and very large data-sets are often limited or insufficient.
* Lacking efficiency: When a content-based search is possible, it can't be used very regularly (for example, for every query) due to routine rationales and because of divergent application.

* Semantics of multimedia data: Derive and Interpreted data, which may be considered as a part of metadata; as well as context and semantics (which may be easier to base on metadata rather than on raw data) are of larger value when working with multimedia data (especially audio-visual data).

\section{Metadata Classification}

The classification of metadata to get very suitable concepts aids in exploring metadata. Thus, metadata can be classified based on [1]:

\section{a) Dependence on content classification}

* Content-independent metadata: This type details information that does not depend on the content of the document with which it is associated with. Examples of this type are location, the type-of-sensor used to record it and modification-date of document. There is no information content captured by these metadata but they are still useful for retrieval of documents from their actual physical location.

* Content-dependent metadata: This type depends on the content of the document it is associated with. Examples of content dependent metadata are maxcolours, size of a document, number of rows and columns in an image. Content-dependent metadata can be sub-divided further into: -

- Direct content-based metadata: This type is based directly on the contents of a document. A popular example of this is full-text indices based on the text of the documents. Inverted tree and document [Christos Faloutsos] vectors are examples of this type of metadata.

- Content descriptive metadata: This type illustrates the content of document without direct utilization of those contents. It often involves the use of knowledge or human perception/cognition. An example of this is the textual annotation that describes the contents of an image. This type come in two flavours:

1) Domain-independent metadata: these metadata capture information present in the document which is independent of the application or subject domain of information. Examples of these are the $\mathrm{C} / \mathrm{C}++$ parse trees and HTML document type definition.

2) Domain-specific metadata: this type metadata is described in a manner specific to the application or subject domain of information. Examples of such metadata are land-cover from GIS and population from Census domain. In case of structural data, the database schema is an example of such metadata. Another example is domain specific ontologies, terms from which may be used to construct metadata specific to domain.

\section{b) Hierarchical Based Classification}

This is another type of classification of metadata that is possible as proposed by Gilliland-Swetland (1998): [18]. This classification includes: 
* Descriptive: are metadata that are used to identify or describe information resources.

* Technical: are metadata that are related to how a system functions or metadata behaves.

* Administrative: are metadata that are used in managing and administering information resources.

* Preservation: are metadata that are related to the preservation management of information resource.

\section{Source of Metadata}

Metadata can be extracted from various sources that are available from the system. Thus, the four main categories [12] of metadata sources are:

a) Document content analysis: an obvious source for metadata about an object is the object itself. An object based indexer generates metadata using the object independent from some precise usage. Typical content analyzers are keyword extractors, language analyzers for text documents or pattern recognizers for images [1].

b) Composite documents structure: In a number of cases, learning objects are parts of a whole but are stored separately. In such a case, the metadata available for the whole is an interesting source for metadata about a component. Not only is the enclosing object a source, also the sibling components can provide relevant metadata. For example, one slide in a slide show often gives relevant context about the content of the next slide. This could be considered as a special case of document context, called "aggregation related context".

c) Document usage: the real use of objects can provide us with more flexible and lively metadata than the sometimes more "theoretical" values that are provided by other metadata sources, or even by human indexers. Systems that track and log the actual use of documents by learners are therefore a priceless source. These logs for example store the time spent reading a document or solving exercises. This metadata source category could be considered as a "usage context", and as such as a special case of document context analysis.

d) Document context analysis: when an object is used in a specific context and data about that context are available, we can rely on the context to obtain information about the object itself. One single learning object typically canbe deployed in several contexts which provide us with metadata about it.

\section{Generation of Metadata}

In the case of structured databases, the standard is to use a schema report and associated information, such as the database statistics as metadata, while in the case of unstructured textual data and information retrieval, metadata is generally limited to indexes and textual descriptions of such data. Metadata in such cases provides a suitable basis for building the higher forms of information. Metadata is commonly generated using three methods [9]

a) Analyzing raw data: In several cases, media objects are analyzed and metadata is created according to the focus of analysis. This is also known as explicit metadata generation.

b) Semi-automatic augmentation: media results in addition to meta-information, which cannot be obtained from the raw material as such. Examples are the diagnostic findings of a doctor related to computer tomography image, which are based on doctor's experience and state of art in medicine.

c) Processing with implicit metadata generation: metadata can be generated implicitly when creating raw media data. For example, the digital camera can implicitly deliver time and date for picture and video taken. Comparably, an SGML editor generates metadata according to document type definition when the document is edited. Generating the metadata can easily be a tiresome task, although using automatic tools may help. The task is more daunting when attempting to generate a vast volume of metadata, lacking the knowledge of the data, its usage, its background facts, as well as its accuracy, etc. Before generating the metadata, it is necessary to review all the relevant documentation about the data.

\section{Metadata standards}

Standards are an important mean to achieve common representation designs and interoperability of a system, and hence it can play a key role in exploiting metadata [19]. There are very many activities that goes on area including:

* The development of metadata taxonomy to help structure the discourse on metadata.

* The definition of generic functionality for tools for the development and operation of metadata base.

* The definition of a meta-model registry structure to achieve mapping among different meta-model, and

* The development of ontology related to metadata attribute and the description of data elements and domains in terms of the naming, typing, classification and the semantics.

\section{IT SERVICE MANAGEMENT AND MULTIMEDIA TECHNOLOGIES}

It is now widely established that the provision of services and it acceptance should be administered by an agreement. This is necessary in order to define the parameters of the service, for the benefit of both the provider and the recipient of such service. It must obviously cover many other issues, as well as defining the service itself. Sadly, the creation of an appropriate and focused Service Level Agreement (SLA) which in itself is NOT a trivial task can be aided by multimedia technologies. An SLA is a part of a service pact where a service is formally defined. In practice, the term SLA is sometimes applied to refer to the agreed delivery time (of the service or performance). [22] For an example, an internet service provider (isp) will commonly include SLAs within the terms of their contracts with the customers to define the various level(s) of the service that is being sold in plain language terms. In this case, the Service Level Agreement will typically have a technical meaning in terms of mean time to repair or the mean time to recovery (MTTR), the mean time between failures (MTBF) as well as the various data rates, the throughput or any other similar measurable details.

\section{IT System support}

Efforts on various fronts have concentrated on the issue of service management, including research on resource scheduling, operating system support for Quality of Service (QoS), the use of multilevel and user-level threads, etc. Other characteristics of multimedia, such as the huge data volumes, may mandate special constraints on the system in terms of the memory management, CPU performance, throughput, and so on. Allied issues include general considerations on input/ output (I/O) hardware to support the various media types 
involved that are involved in multimedia databases. Communication networks - that are required to convey the data for distributed multimedia environments - must support the bandwidth and delay guarantees as needed to meet the stringent QoS requirements for certain multimedia applications.

\section{Applications}

Broadly speaking, multimedia database management systems applications can be established wherever there is a need to manage multimedia data cost-effectively. Therefore multimedia DBMSs have found applications in such varied areas as healthcare (telemedicine, integrated health information management, medical image systems), education (digital libraries, training, presentation, distance learning), entertainment (video-on-demand, music databases, interactive TV), information dissemination (news-on-demand, advertising, TV broadcasting), and manufacturing (distributed manufacturing, distributed collaborative authoring). Such other areas of its application may consist of finance, ecommerce, electronic publishing, geographic information systems, video conferencing, etc [20].

A number of multimedia DBMSs are already in existence. Most of them are annexes from existing relational or objectoriented DBMSs. The competence of existing multimedia DBMSs can be appraised by the extent to which they can support different media types (especially image and video). They can also be evaluated by their ability to support special functionalities required of a database system to manage multimedia data, such as real-time delivery and content based query and retrieval [15]. Regrettably, most existing multimedia DBMSs fall short of this. Nonetheless, rigorous research is variously being geared toward the various parts of the problem and as such it is expected that new systems with higher and better capabilities in the near future will emerge. Future trends include performing indexing, retrieval, and browsing directly on the compressed data, especially for video data; video data management; multimedia query language; uniform indexing frameworks for the different data types; content-based image and video retrieval; and multimedia transport and delivery over the Internet [12].

\section{CONCLUSION}

TheMultimedia data should be stored with advanced data structures and with the aid of metadata in order to make it search and retrieval process simpler. Thus an approach on how images can be stored using data structures and can be searched has been examined. This data structure helps to remove attributes from data like image or video so that we can execute content based queries. Also the advantage and disadvantage of these data structures were discussed. However, sometimes the indexing and searching process consume lots of time in case of large databases [21]. So we need help of metadata to make that process faster which does not require extracting characteristics and information from the data itself. Also presented is how metadata is generated and it mentioned several issue that have to be undertaken. Also how metadata can be classified so that depending upon the context, better use of it can be made. Also how metadata standards can assist exploiting use of metadata. In general, multimedia applications and distributed multimedia database systems especially, raise some new issues in all aspects of the computer system, these ranges from operating systems to networks to the general hardware [16]. Broadly available operating systems do not support real-time operations sufficiently. Somewhat, they offer hardware front-ends for conveying and presenting multimedia data. Some multimedia data, such as continuous media, may require real-time delivery and presentation, while the real-time requisites might not be as rigid as those encountered in hard real-time systems. Therefore, the multimedia database system cannot fully provide its functionalities until support for real-time continuous media data becomes an integral part of the operating system.

\section{ACKNOWLEDGMENTS}

I acknowledge the original and earlier works of Ajit Burad (Multimedia Databases, Computer Science and Engineering, Indian Institute of Technology, April 6, 2006, Donald A. Adjeroh and Kingsley C. Nwosu (Multimedia Database Management - Requirements and Issues) as well as Milko Marinov and Dimitar Radev (An Implementation of a Multimedia Object-Oriented Database Management System).

\section{REFERENCES}

[1] Ajit Burad, "Multimedia Databases", Computer Science and Engineering, Indian Institute of Technology, April 6, 2006

[2] Antonm Guttman, "R-trees: A dynamic index structure for spatial database" Proceedings ACM SIGMOD Conference, pp.47-57, Boston, MA, 1984.

[3] Arjen P. De Vries, "Content and Multimedia database Management Systems" Centre for Telematics and Information Technology (CTIT), The Netherlands

[4] Christos Faloutsos, "Indexing Multimedia Database" Proceedings of the 1995 ACM SIGMOD International Conference on Management of Data, San Jose, California, May 22-25,

[5] Danail Dochev, Irena Koprinska and Radoslav Pavlov, "Multimedia Data Management - Characteristics and Requirements" Institute of Information Technologies, 2000, 1113 Sofia

[6] Donald A. Adjeroh, Kingsley C. Nwosu, Multimedia Database Management - Requirements and Issues". IEEE, 2007.

[7] F.G.B. De Natale and F. Granelli, "Structure-Based Image retrieval using as structured colour descriptor" Workshop on Content-Based Multimedia Indexing (CBMI’01), pages 109-115, Brescia (Italy), Sept. 2001.

[8] Gunasekaran, P.E.D. Love, "Current and future directions of multimedia technology in business" International Journal of Information Management 19 (1999) $105-120$

[9] Jane Greenberg, Metadata Extraction and Harvesting, "A Comparison of Two Automatic Metadata Generation Applications". Journal of Internet Cataloguing, 6(4): 5982 .

[10] JI-Rong Wen, Qing Li, Wei-Ying Ma, Hong-jiang Zhang, "A Multi-paradigm Querying Approach for a Generic Multimedia Database Management System" SIGMOD Record, Vol. 32, No. 1, March 2003

[11] Joseph Kuan and Paul Lewis, Fast k-nearest neighbour search for R-tree family" In Proc. of First Int. Conf. on Information, Communication and Signal Processing, pages 924-928, Singapore, 1997. 2.2 
[12] Kris Cardinels, Michael Meire, Erik Duval, "Automating Metadata Generation: the Simple Indexing Interface" Proceedings of the 14th International Conference on World Wide Web May, 2005.

[13] P. Prabhakaran, "Multimedia Database Management Systems", Kluver Academic Publishers, 2005.

[14] Peter van Oosterom, "Spatial Access Methods Chapter in Geographical Information Systems Principles, Technical Issues, Management Issues, and Applications (edited)"

[15] Reshma Suvarna, K. Seluk Candan1, Huan Liu, Jong Wook Kim, "Structure based Mining of Hierarchical Media Data, Meta-Data, and Ontologies" The 5th International Workshop on Multimedia Data Mining (MDM/KDD2004)

[16] Robert Garcia and Oscar Celma, "Semantic Integration and Retrieval of Multimedia Metadata" Proceedings of the 5th International Workshop on Knowledge Markup and Semantic Annotation (SemAnnot 2005) to be held with ISWC 2005, Galway, Ireland, 7 November 2005
[17] Sherry Marcus and V.S. Subrahmanian, "Foundation of multimedia database system" Volume 43 Journal of ACM (May 1996)

[18] Sussane Boll, Wolfgang Klas and Amit Sheth "Overview on using Metadata to Manage Multimedia Data book" Multimedia Data Management pages 1-24

[19] V. S. Subrahamaniam, "Principles of Multimedia Database System” Morgan Kaufmann Publishers, 1998.

[20] Walid G. Aref and Ihab F. Ilyas, "An extensible index for spatial database". Proceedings of the 13th International Conference on Scientific and Statistical Database Management, July 18-20, 2001, George Mason University, Fairfax, Virginia, USA

[21] Yu Deng, "The Metadata Architecture for Data Management in Web-based Choropleth Maps" Department of Computer Science, University of Maryland.

[22] Yuchai Zhou, "An approach to building Metadata for Geo-referenced Multimedia Data" GEOG 5905: Master's Research Workshop, Carleton University. 